\title{
Control and optimal operation of simple heat pump cycles
}

\author{
Jørgen B. Jensen and Sigurd Skogestad* \\ Department of Chemical Engineering, NTNU \\ N-7491 Trondheim, Norway
}

\begin{abstract}
Cycles for heating and cooling have traditionally been studied in detail when it comes to thermodynamics and design. However, there are few publications on their optimal operation which is the theme of this paper. One important issue is which variable to control, for example, degree of super-heating, pressure, liquid level or valve opening. Also, unlike open systems, the initial charge to the cycle may have a steady state effect, and it is discussed how different designs are affected by this factor. Numerical results are provided for an ammonia cycle.
\end{abstract}

Keywords: Operation, heat pump cycle, cyclic process, charge, self-optimizing control

\section{Introduction}

Cyclic processes for heating and cooling are widely used in many applications and their power ranges from less than $1 \mathrm{~kW}$ to above $100 \mathrm{MW}$. All of these applications use the vapor compression cycle to "pump" energy from a low to a high temperature level.

A schematic drawing of a simple cycle is shown in figure 1 together with a typical pressure-enthalpy diagram for a sub-critical cycle. The way the cycle works:

The low pressure vapor (4) is compressed by supplying work $W_{s}$ to give a high pressure vapor with high temperature (1). This stream is cooled to the saturation temperature in the first part of the condenser, condensed in the middle part and possibly sub-cooled in the last part to give the liquid (2). In the expansion choke, the pressure is lowered to its original value, resulting in a two-phase mixture (3). This mixture is vaporized and heated through the evaporator giving a super-heated vapor (4) closing the cycle.

The coefficients of performance for a heating cycle (heat pump) and a cooling cycle (refrigerator, A/C) are defined as

$C O P_{h}=\frac{Q_{h}}{W_{s}}=\frac{h_{1}-h_{2}}{h_{1}-h_{4}} \quad$ and $\quad C O P_{c}=\frac{Q_{c}}{W_{s}}=\frac{h_{4}-h_{3}}{h_{1}-h_{4}}$

respectively. Heat pumps typically have a COP of around 3 which indicates that $33 \%$ of the gained heat is addet as work (eg. electric power).

In industrial processes, especially in cryogenic processes such as air separation and liquefaction of natural gas (LNG process), more complex cycles are used in order to improve the thermodynamic efficiencies. These modifications lower the temperature differences in the heat exchangers and include cycles with mixed refrigerants, several pressure levels and cascaded cycles. The Mixed Fluid Cascade process developed by the Statoil Linde

*Author to whom correspondence should be addressed: skoge@ chemeng.ntnu.no 

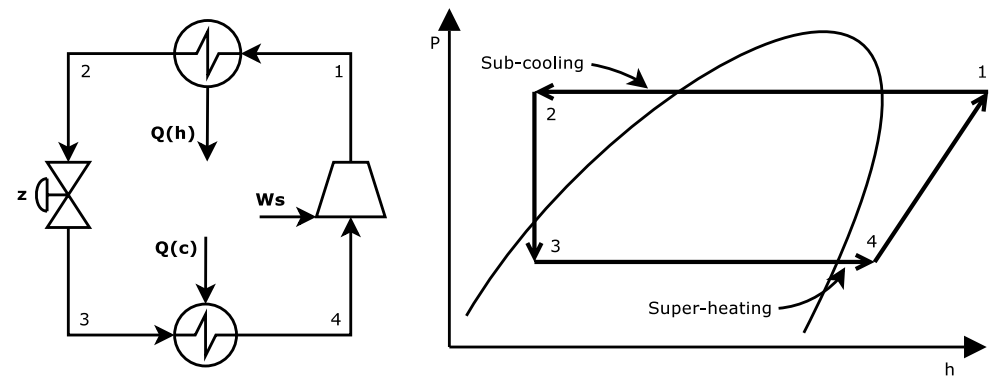

Figure 1. Schematics of a simple vapor compression cycle with typical pressure-enthalpy diagram

Table 1. Specifications in design and operation

\begin{tabular}{llc} 
& Given & $\#$ \\
\hline Design & Load (e.g. $\left.Q_{h}\right), P_{l}, P_{h}, \Delta T_{\text {sup }}$ and $\Delta T_{\text {sub }}$ & 5 \\
Operation & $W_{s}$ (load), choke valve opening (z) and $U A$ in two heat exchangers & 4
\end{tabular}

Technology Alliance is being built at the LNG plant in northern Norway and incorporates all of the above modifications. The resulting plant has three cycles, all with mixed refrigerant and the first with two pressure levels. Our long term objective is to study the operation of such processes. However, as a start we need to understand the simple cycle in figure 1.

\section{Operation of simple vapor compression cycles}

\subsection{Design versus operation}

Table 1 shows typical specifications for simple cycles in design (find equipment) and in operation (given equipment). Note that the five design specifications results in only four equipment parameters; compressor work $W_{s}$, valve opening $\mathrm{z}$ and UA for the two heat exchangers. As a consequence, with the four equipment parameters specified, there is not a unique solution in terms of the operation. The "un-controlled" mode is related to the pressure level, which is indirectly set by the charge of the system. This is unique for closed systems since there is no boundary condition for pressure. In practice, the "pressure level" is adjusted directly or indirectly, depending on the design, especially of the evaporator. This is considered in more detail below.
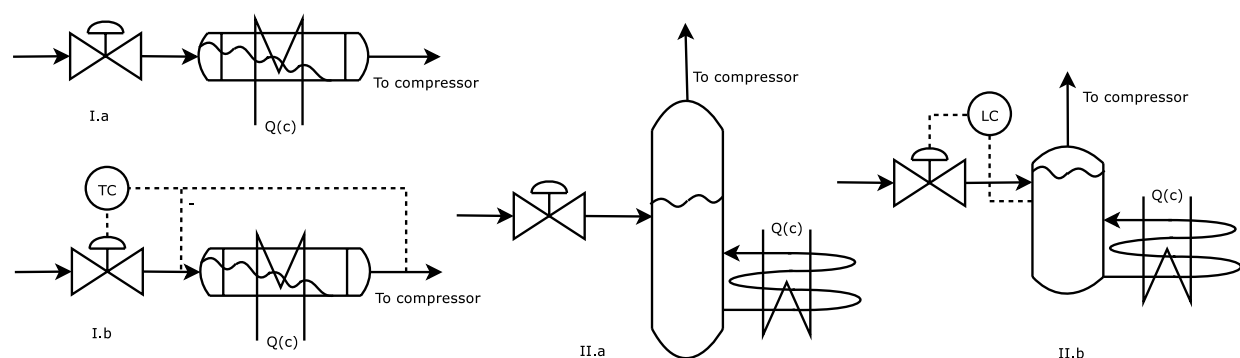

Figure 2. Four different evaporator designs; I. a Dry evaporator, I.b with TEV, II.a Flooded evaporator, II.b with level control 


\subsection{Operational (control) degrees of freedom}

During operation the equipment is given. Nevertheless, we have some operational or control degrees of freedom. These include the compressor power $\left(W_{s}\right)$, the charge (amount of vapor and liquid in the closed system), and the valve openings. The following valves may be adjusted on-line:

- Adjustable choke valve (z); see figure 1 (not available in some simple cycles)

- Adjustable valve between condenser and storage tank (for designs with a separate liquid storage tank before the choke; see design III.a in figure 3)

In addition, we might install bypass valves on the condenser and evaporator to effectively reduce UA, but this is not normally used because use of bypass gives suboptimal operation.

Some remarks:

- The compression power $W_{s}$ sets the "load" for the cycle, but it is otherwise not used for optimization, so in the following we do not consider it as an operational degree of freedom.

- The charge has a steady-state effect for some designs because the pressure level in the system depends on the charge. A typical example is household refrigeration systems. However, such designs are generally undesirable. First, the charge can usually not be adjusted continuously. Second, the operation is sensitive to the initial charge and later to leaks.

- The overall charge has no steady-state effect for some designs. This is when we have a storage tank where the liquid level has no steady-state effect. This includes designs with a liquid storage tank after the condenser (III.a, figure 3), as well as flooded evaporators with variable liquid level (II.a, figure 2). For such designs the charge only effects the level in the storage tank. Note that it may be possible to control (adjust) the liquid level for these designs (II.a, figure 2), and this may then be viewed as a way of continuously adjusting the charge to the rest of the system (condenser and evaporator).

- There are two main evaporator designs; the dry evaporator (I) and the flooded evaporator (II) shown in figure 2. In a dry evaporator, we generally get some superheating, whereas there is no (or little) super-heating in a flooded evaporator. The latter design is better thermodynamically, because super-heating is undesirable from an efficiency (COP) point of view. In a dry evaporator one would like to control the super-heating, but this is not needed in a flooded evaporator. In addition, as just mentioned, a flooded evaporator with variable liquid level is insensitive to the charge.

- It is also possible to have flooded condensers. and thereby no sub-cooling, but this is not desirable from a thermodynamic point of view.

\subsection{Use of the control degrees of freedom}

In summary, we are during operation left with the valves as degrees of freedom. These valves should generally be used to optimize the operation, In most cases "optimal operation" is defined as maximizing the efficiency factor, COP. We could then envisage an 
on-line optimization scheme where one continuously optimizes the operation (maximizes COP) by adjusting the valves. However, such schemes are quite complex and sensitive to uncertainty, so in practice one uses simpler schemes where the valves are used to control some other variable. Such variables could be:

- Valve position setpoint $z_{s}$ (that is, the valve is left in a constant position)

- High pressure $\left(P_{h}\right)$

- Low pressure $\left(P_{l}\right)$

- Temperature out of condenser $\left(T_{2}\right)$ or degree of sub-cooling $\left(\Delta T_{\text {sub }}=T_{2}-T^{\text {sat }}\left(P_{h}\right)\right)$

- Temperature out of evaporator $\left(T_{4}\right)$ or degree of super-heating $\left(\Delta T_{\text {sup }}=T_{4}-T^{\text {sat }}\left(P_{l}\right)\right)$

- Liquid level in storage tank (to adjust charge to rest of system)

The objective is to achieve "self-optimizing" control where a constant setpoint for the selected variable indirectly leads to near-optimal operation (Skogestad, 2000).

Control (or rather minimization) of the degree of super-heating is useful for dry evaporators (with TEV, design I.b). However, it consumes a degree of freedom. In order to retain the degree of freedom, we need to add a liquid storage tank after the condenser (design III.a). In a flooded evaporator, the super-heating is minimized by design so no control is needed.

With the degree of super-heating fixed (by control III.a or design II.a), there is only one degree of freedom left that needs to be controlled in order to optimize COP. To see this, recall that there are 5 design specifications, so optimizing these give an optimal design. During operation, we assume the load is given $\left(W_{s}\right)$, and that the maximum areas are used in the two heat exchangers (this is optimal). This sets 3 parameters, so with the super-heating controlled, we have one parameter left that effects COP.

In conclusion, we need to set one variable, in addition to $\Delta T_{\text {sup }}$, in order to completely specify (and optimize) the operation. This variable could be selected from the above list, but there are also other possibilities. Some common control schemes are discussed in the following.

\subsection{Some alternative designs and control schemes}

Some designs are here presented and the pro's and con's are summarized in table 2 .

\subsubsection{Dry evaporator (I)}

For this design there is generally some super-heating.

I.a In residential refrigerators it is common to replace the valve by a capillary tube, which is a small diameter tube designed to give a certain pressure drop. On-off control of the compressor is also common, but we will consider capacity control to get comparable results.

I.b Larger systems usually have a thermostatic expansion valve (TEV), (Dossat, 2002) and (Langley, 2002), that controls the temperature and avoids excessive super-heating. A typical super-heat value is $10^{\circ} \mathrm{C}$.

\subsubsection{Flooded evaporator (II)}

A flooded evaporator differs from the dry evaporator in that it only provides vaporization and no super-heating. 


\begin{tabular}{|c|c|c|}
\hline & Pro's & Con's \\
\hline I.a & Simple design & $\begin{array}{l}\text { Sensitive to charge } \\
\text { No control of super-heating }\end{array}$ \\
\hline I.b & Controlled super-heating & $\begin{array}{l}\text { Super-heating } \\
\text { Sensitive to charge }\end{array}$ \\
\hline II.a & $\begin{array}{l}\text { No super-heating by design } \\
\text { Not sensitive to charge }\end{array}$ & \\
\hline & Valve is free & How to use valve? \\
\hline II.b & No super-heating by design & Sensitive to charge \\
\hline III.a & Not sensitive to charge & $\begin{array}{l}\text { Complex design } \\
\text { How to use valve? }\end{array}$ \\
\hline
\end{tabular}

II.b In flooded evaporator systems the valve is used to control the level in either evaporator or condenser (figure 2 II.b).

II.a We propose a design where the volume of the flooded evaporator is so large that there is no need to control the level in one of the heat exchangers. This design retains the valve as a degree of freedom.

\subsubsection{Other designs (III)}

III.a To reduce the sensitivity to the charge in designs I.b and II.b it is possible to include a liquid receiver before the valve as shown in figure 3 . To retain a degree of freedom a valve may be added before the receiver (shown with dashed lines).

III.b It is possible to add a internal heat exchanger as shown in figure 3. This will superheat the vapor entering the compressor and sub-cool the liquid before expansion. The latter is positive because of reduced expansion losses, whereas the first is undesirable because compressor power increases.
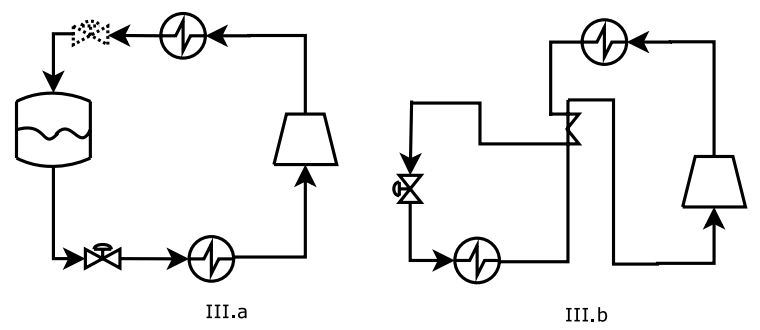

Figure 3. Special design features; III.a Liquid receiver, III.b Internal heat exchanger

\section{Ammonia case study}

Figure 4 shows a pressure enthalpy diagram for the ammonia case study. The cycle operates between $-10^{\circ} \mathrm{C}$ and $20^{\circ} \mathrm{C}$ taking out $20 \mathrm{~kW}$ of heat. The model and diagram is based on simplified thermodynamics with constant heat capacities. A detailed model description and more results are available from the internet (Jensen and Skogestad, 2005). In table 3 we show the effect of super-heating. Designs I.a and I.b (with TEV) require some superheating to assure vapor out of evaporator, whereas the flooded evaporator (II.a/b) has no super-heating. The result is an improvement in $\mathrm{COP}_{C}$ from $4.56\left(10^{\circ} \mathrm{C}\right.$ super-heating $)$ to 5.39 . 
Table 3. The influence of super-heating on performance for given equipment and load

\begin{tabular}{cc}
$\Delta T_{\text {sup }}\left[{ }^{\circ} \mathrm{C}\right]$ & $C O P_{c}$ \\
\hline 10 & 4.56 \\
0 & 5.39
\end{tabular}

The main disturbance for the cycle is the air temperature in the condenser $\left(T_{\text {air }}\right)$ and we now consider a case where $T_{\text {air }}$ increases by $10{ }^{\circ} \mathrm{C}$ while the load $\left(Q_{c}\right)$ remains constant. Table 4 shows for alternative controlled variables the losses in terms of increased work $W_{s}$ compared with a re-optimized case. This is done for design II with no super-heating where there is one degree of freedom. Pressure control gives infeasible operation. Fixing the valve gives a loss of about $12 \%$ and fixing the evaporator level (design II.b) gives a loss $1.6 \%$. The best "self-optimizing" controlled variables are the condenser liquid level and the condenser sub-cooling $\left(\Delta T_{s u b}\right)$ which have losses of only $0.02 \%$. A more detailed study reveals that constant condenser level is the best strategy (not considering implementation accuracy).

Table 4. Loss for different control structures for $10^{\circ} \mathrm{C}$ increase in $T_{\text {air }}$ (design II with constant $Q_{c}$

\begin{tabular}{ccccccc} 
Constant & $\mathrm{z}$ & $P_{h}$ & $P_{l}$ & Evaporator level & Condenser level & $\Delta T_{\text {sub }}$ \\
\hline$\Delta W_{s}[\%]$ & 11.8 & Inf $^{a}$ & $\operatorname{Inf}^{b}$ & 1.6 & 0.02 & 0.02
\end{tabular}

\footnotetext{
Inf $=$ Infeasible

${ }^{a}$ Feasible only for small disturbances

${ }^{b}$ Not possible to fix both evaporator- duty $\left(Q_{c}\right)$ and pressure $\left(P_{l}\right)$ since $Q_{c}=U A\left(T^{\text {sat }}\left(P_{l}\right)-T_{\text {air }}\right)$
}

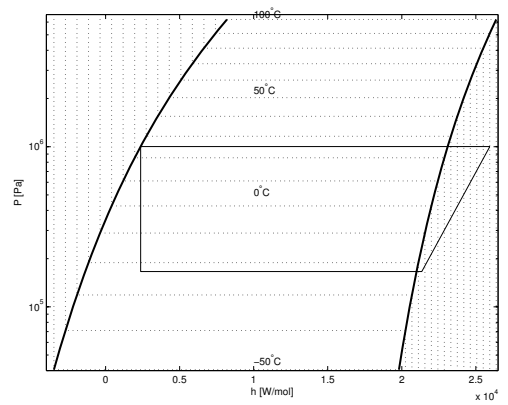

Figure 4. Pressure-enthalpy diagram for optimal design of the ammonia case study using design I.b

\section{References}

Dossat, R. J. (2002). Principles of refrigeration. Prentice Hall.

Jensen, J. B. and Skogestad, S. (2005). Study of an Ammonia vapor compression cycle, Internal report. http://www.nt.ntnu.no/users/skoge/publications/2005/jensen_ internal_report/.

Langley, B. C. (2002). Heat pump technology. Prentice Hall.

Skogestad, S. (2000). Plantwide control: the search for the self-optimizing control structure. Journal of Process Control, 10(5):pp. 487-507.

\section{Acknowledgments}

This work has been done as part of the The Gas Technology Center, NTNU-SINTEF. Funding from the Norwegian Research Council is gratefully acknowledged. 